\title{
Índice de FRAIL como predictor de complicaciones postquirúrgicas en pacientes ancianos
}

\author{
FRAIL index as a predictor of post-surgical \\ complications in elderly patients
}

\author{
Eric Misael Saucedo Moreno, ${ }^{*}$ Enrique Fernández Rivera, ${ }^{*}$ \\ José Abenamar Ricárdez García, * Laura Elisa García y Moreno, ${ }^{\neq}$ \\ Fabián Gaona Reyes, ${ }^{\S}$ María Fernanda Chimal Juárez
}

Citar como: Saucedo MEM, Fernández RE, Ricárdez GJA, García y Moreno LE, Gaona RF, Chimal JMF. Índice de FRAIL como predictor de complicaciones postquirúrgicas en pacientes ancianos. Acta Med Grupo Angeles. 2021; 19 (3): 327-332. https://dx.doi.org/10.35366/101723

\section{Resumen}

Objetivo: Identificar si el índice de FRAIL tiene utilidad como predictor de complicaciones postquirúrgicas en pacientes mayores de 65 años. Material y métodos: Estudio descriptivo, transversal de una cohorte de 60 pacientes mayores de 65 años operados por padecimientos agudos. A todos se les aplicó el índice de FRAIL previo al evento quirúrgico para identificar a los pacientes frágiles de los no frágiles, evaluamos y comparamos las complicaciones y la mortalidad entre cada grupo de manera objetiva con la prueba índice de complicaciones integral ( $\mathrm{CCl}$, por sus siglas en inglés). Resultados: La media del índice de FRAIL preoperatorio fue de $1.82 \pm 0.983$. De los pacientes, $21.6 \%$ fueron considerados frágiles y $41.7 \%$ tuvieron complicaciones graves. Identificamos que existe un riesgo cuatro veces más elevado de presentar complicaciones mayores $(\mathrm{CCl} \geq 25)$ en pacientes con fragilidad vs. pacientes no frágiles, OR = 4.479 (IC 95\% 1.13 a 17.73, $p=0.033$ ). Conclusión: El índice de FRAIL es una herramienta fácil de utilizar para identificar de manera oportuna a pacientes que tienen síndrome de fragilidad, quienes tienen un riesgo cuatro veces mayor de complicaciones postquirúrgicas.

Palabras clave: Fragilidad, cirugía, complicaciones postoperatorias, mortalidad.

\section{Abstract}

Objective: Identifying if the FRAIL index is useful as a predictor of post-surgical complications in patients other than 65 years. Material and methods: We studied a sample of 60 patients older than 65 years operated for acute conditions. We all applied the FRAIL index prior to the surgical event to identify those patients who are fragile and non-fragile. We evaluate and compare complications and mortality between each group by objectively with the comprehensive complication index $(\mathrm{CCl})$ test. Results: The mean preoperative FRAIL index was 1.82 \pm 0.983 . The $21.6 \%$ were fragile. The $41.7 \%$ of the patients had serious complications. We identified that there is a four times higher risk of presenting major complications (ICC $\geq 25)$ in patients with fragility vs non-fragile patients, $O R=4.479$ $(95 \% \mathrm{Cl} 1.13$ to $17.73, p=0.033)$. Conclusion: The FRAIL index is an easy and usable tool to identify those with fragility syndrome in a timely manner, who are at four times the risk of post-surgical complications.

Keywords: Frailty, surgery, postoperative complications, mortality.
* Cirujano General. Servicio de Cirugía General. Hospital Ángeles Mocel. México.

₹ Médico residente de cuarto año de Cirugía General. Facultad Mexicana de Medicina de la Universidad La Salle. México.

$\S$ Médico residente de tercer año de Cirugía General. Facultad Mexicana de Medicina de la Universidad La Salle. México.

" Médico residente de segundo año de Cirugía General. Facultad Mexicana de Medicina de la Universidad La Salle. México.

\section{Correspondencia:}

Eric Misael Saucedo Moreno

Correo electrónico: eric.saucedo.m@gmail.com

Aceptado: 09-09-2020.

www.medigraphic.com/actamedica

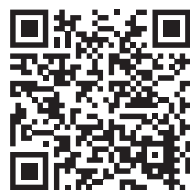




\section{INTRODUCCIÓN}

La Organización Mundial de la Salud (OMS) informó en 2019 que existen 703 millones de personas mayores de 60 años y proyecta que para 2050 el número de personas ascenderá a 1,500 millones. ${ }^{1}$ De acuerdo al reporte nacional de pacientes ancianos en Estados Unidos, la proporción de adultos mayores de 65 años se encontraba en $4.1 \%$ en 1900 , cerca de $13.7 \%$ en el 2021 y se encuentra proyectado un incremento al $21 \%$ para el año 2040 . El INEGI reporta en la última encuesta de 2018: el número de personas mayores de 60 años en México es de 15.4 millones, cifra que representa $12.3 \%$ de la población total. ${ }^{2}$

Un gran número de estos pacientes ingresan a los servicios de cirugía para ser operados de manera programada y estudiada, con la intención de disminuir los riesgos de complicaciones postoperatorias; por el contrario, en pacientes con patologías agudas no existe una forma de pronosticar y reconocer el riesgo de estas complicaciones e incluso la mortalidad. ${ }^{3-5}$ Varios estudios han demostrado que la edad no es directamente proporcional al riesgo de complicaciones postquirúrgicas, más bien esta vulnerabilidad se encuentra ligada a la disminución de la reserva y la función de múltiples sistemas fisiológicos asociada al envejecimiento, que comprometen la capacidad de lidiar con estresores cotidianos y/o agudos, siendo posible

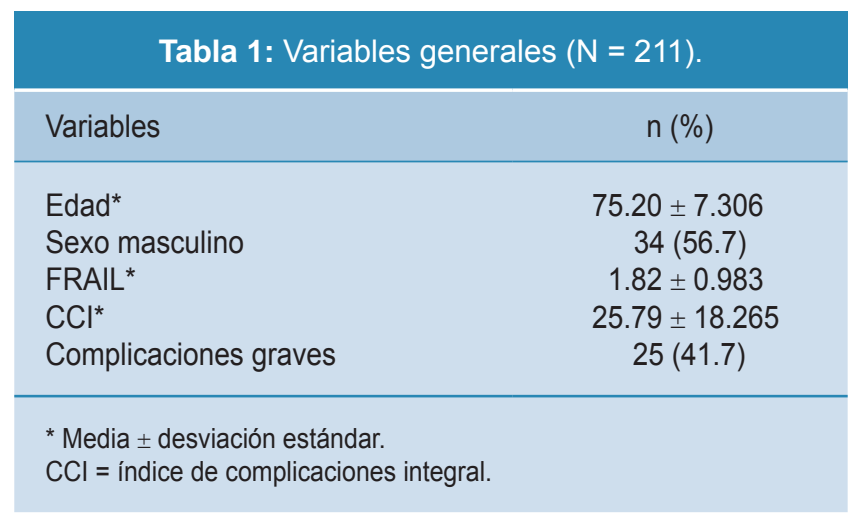

reconocer clínicamente como síndrome de fragilidad. ${ }^{6,7} \mathrm{El}$ índice de FRAIL es una herramienta validada y recomendada como cribado para identificar personas frágiles que, de ser positiva, orilla a realizar un estudio más profundo de la enfermedad. El objetivo de este estudio es identificar la utilidad que tiene el índice de FRAIL para predecir complicaciones postquirúrgicas en pacientes ancianos comparando los pacientes frágiles de los no frágiles. ${ }^{8}$

\section{MATERIAL Y MÉTODOS}

Realizamos un estudio observacional, descriptivo en un hospital privado en la Ciudad de México de manera prospectiva en un periodo de un año y ocho meses, comprendido entre septiembre de 2017 y diciembre de 2019. Incluimos a todos los pacientes ancianos (mayores de 65 años) que fueron ingresados al hospital por alguna patología abdominal aguda y que ameritaron resolución quirúrgica o aquéllos que se encontraban en hospitalización y presentaron dolor abdominal que ameritó tratamiento quirúrgico. No se incluyeron pacientes con cirugías torácicas, vasculares o neurológicas. Se excluyeron los pacientes que se encontraban ingresados en la unidad de terapia intensiva previo al evento quirúrgico y aquéllos en los que no fue posible obtener los datos completos del índice de FRAIL previo al evento quirúrgico. A todos los pacientes les aplicamos el test FRAIL al entrar al protocolo de estudio (durante su estancia en urgencias o previo al evento quirúrgico si se encontraban hospitalizados) para calcular el índice de fragilidad, el cual consiste en cinco preguntas. Clasificamos la muestra en dos grupos de acuerdo al puntaje obtenido. El grupo 1 representa a los pacientes frágiles con valores $\geq 3$ puntos y el grupo 2 a los no frágiles, aquéllos con valores $\leq 2$ puntos. Dimos un seguimiento de las complicaciones durante su estancia intrahospitalaria con la clasificación $\mathrm{CCl}$, la cual otorga un puntaje de acuerdo con la gravedad de las complicaciones y al número de las mismas, a quienes obtuvieron puntajes $\geq 25$ los consideramos como complicaciones graves.
¿Está usted cansado?

¿Es incapaz de subir un piso de escaleras?

¿Es incapaz de caminar una manzana?

¿Tiene más de cinco enfermedades?

¿Ha perdido más de $5 \%$ de su peso en los últimos seis meses?
$32(53.3)$

$34(56.7)$

$12(20.0)$

$23(38.3)$

$10(16.7)$ 
Tabla 3: Comparación de variables entre ambos grupos.

\begin{tabular}{|c|c|c|c|}
\hline & Frágiles, $\mathrm{N}=13(21.6 \%)$ & No frágiles, $\mathrm{N}=47(78.4 \%)$ & \\
\hline & $\mathrm{n}(\%)$ & $\mathrm{n}(\%)$ & $\mathrm{p}^{*}$ \\
\hline Edad (rango) & $75(66$ a 92$)$ & $74(65$ a 88$)$ & 0.133 \\
\hline $\mathrm{CCl}$ total (rango) & $28.5(12$ a 63$)$ & $12(9$ a 63$)$ & 0.017 \\
\hline ¿Está usted cansado? & $11(84.6)$ & $21(44.7)$ & 0.004 \\
\hline ¿Es incapaz de subir un piso de escaleras? & $13(100)$ & $21(44.7)$ & 0.000 \\
\hline ¿Es incapaz de caminar una manzana? & $6(46.2)$ & $6(12.8)$ & 0.044 \\
\hline ¿Tiene más de cinco enfermedades? & $9(69.2)$ & $14(29.8)$ & 0.016 \\
\hline ¿Ha perdido más de $5 \%$ de su peso en los últimos seis meses? & $6(46.2)$ & $4(8.5)$ & 0.025 \\
\hline Complicaciones mayores $(\mathrm{CCl} \geq 25)$ & $9(69.2)$ & $15(31.9)$ & 0.024 \\
\hline Sexo masculino & $7(53.8)$ & $27(57.4)$ & 1.000 \\
\hline Fiebre & $6(46.2)$ & $3(6.4)$ & 0.002 \\
\hline Dolor & $13(100)$ & $45(95.7)$ & 1.000 \\
\hline Edema & $7(53.8)$ & $11(23.4)$ & 0.046 \\
\hline Íleo & $7(53.8)$ & $11(23.4)$ & 0.046 \\
\hline Absceso & 0 & $5(10.6)$ & 0.575 \\
\hline Atelectasia & $1(7.7)$ & $4(8.5)$ & 1.000 \\
\hline Náusea y vómito & $13(100)$ & $28(59.6)$ & 0.005 \\
\hline IVU & $1(7.7)$ & 0 & 0.217 \\
\hline Arritmias & $2(15.4)$ & $1(2.1)$ & 0.115 \\
\hline Neumonía & $3(23.1)$ & 0 & 0.008 \\
\hline Infección de herida & $1(7.7)$ & 0 & 0.217 \\
\hline TVP & $2(15.4)$ & $2(4.3)$ & 0.202 \\
\hline Falla orgánica única & $1(7.7)$ & $1(2.1)$ & 0.389 \\
\hline Falla orgánica múltiple & $1(7.7)$ & 0 & 0.217 \\
\hline UTI & $5(38.5)$ & $6(12.8)$ & 0.049 \\
\hline Nutrición parenteral & $2(15.4)$ & 0 & 0.217 \\
\hline Muerte & $1(7.7)$ & 0 & 0.217 \\
\hline
\end{tabular}

Analizamos las variables con distribuciones paramétricas con la prueba t de Student y se expresaron como media y desviación estándar (DE), mientras que aquéllas con distribuciones no paramétricas fueron analizadas con la prueba $U$ de Mann-Whitney y presentadas como medianas, mínimos y máximos. Utilizamos la prueba $\chi^{2}$ o Fisher para datos categóricos. Realizamos una regresión logística univariada y multivariada considerando un valor de $p=$ 0.05 como estadísticamente significativo y un intervalo de confianza de $95 \%$ (IC). Los datos se analizaron utilizando el software SPSS (versión 26.0 para MAC). ${ }^{9}$

\section{RESULTADOS}

Estudiamos una muestra de 78 pacientes ingresados al hospital mayores de 65 años, operados por padeci- mientos agudos; 18 pacientes fueron excluidos por no contestar completamente las preguntas y/o decidieron abandonar el estudio, con un total de 60 pacientes estudiados. De los participantes, 56.7\% (34) pertenecen al sexo masculino, con una media de edad de $75.2 \pm$ 7.30 años. La media del índice de FRAIL preoperatorio fue de $1.82 \pm 0.983$, y $21.6 \%$ de los pacientes se consideraron frágiles ( $\geq 3$ puntos). Las comorbilidades evaluadas de forma objetiva con la prueba $\mathrm{CCl}$ tuvieron una media de $25.79 \pm 18.265,41.7 \%$ de los pacientes (25) tuvieron complicaciones graves ( $\mathrm{CCl} \geq 25$ puntos) (Tabla 1).

Las variables más frecuentes en el índice de FRAIL fueron la incapacidad para subir escaleras en $56.7 \%$ (34) y la sensación de estar cansado en 53.3\% (32) de los pacientes (Tabla 2). 
Dividimos nuestra muestra en dos grupos. El grupo 1 representa a los pacientes frágiles 13 (21.6\%), y el grupo 2 a los no frágiles $47(78.3 \%)$, al comparar las medianas identificamos que no existen diferencias significativas en la edad para ser considerado frágil ( $p=0.133$, IC 95\% -1.531 a 10.447). Observamos diferencias estadísticamente significativas para complicaciones al obtener valores más altos de $\mathrm{CCl}$ para el grupo de fragilidad vs. no fragilidad, 28.5 vs. 12 respectivamente ( $p=0.017$, IC 95\% 2.89 a 29.77). Las complicaciones con diferencias significativas fueron: fiebre, edema, íleo, náusea y vómito, neumonía y estancia en la unidad de terapia intensiva. Al comparar la mortalidad no identificamos diferencias significativas entre ambos grupos 1 vs. 0 ( $p=0.217$, IC 95\% 21.581 a 38.136) (Tabla 3, Figuras 1 y 2).

Realizamos una regresión logística univariada y multivariada, identificamos que existe un riesgo cuatro veces más elevado de presentar complicaciones mayores $(\mathrm{CCl}>25)$ en pacientes con fragilidad OR $=4.479$ (IC 95\% $1.13 \mathrm{a}$ $17.73, p=0.033$ ). La edad como factor independiente no mostró tener mayor riesgo de complicaciones $\mathrm{OR}=1.018$ (IC 95\% 0.93 a 1.10, p = 0.692) (Tabla 4).

\section{DISCUSIÓN}

En la última década hemos identificado un aumento en la población mayor de 65 años sometida a procedimientos quirúrgicos de urgencia. Varios estudios han demostrado que este grupo de pacientes presentan un riesgo elevado de morbilidad y mortalidad postquirúrgica; esta vulnerabilidad se encuentra ligada a la disminución de la reserva y la función de múltiples sistemas fisiológicos asociada al envejecimiento, reconocida clínicamente como síndrome de fragilidad. Se ha considerado a la fragilidad como una piedra angular en la medicina geriátrica y una plataforma de vulnerabilidad biológica y resultados adversos para la salud. Fried y colaboradores definen la fragilidad como la reunión de tres de los cinco criterios fenotípicos: debilidad, lentitud, agotamiento, baja actividad y pérdida de peso involuntaria; considerando que un sujeto es frágil si cumple tres o más criterios, prefrágil si cumple uno o dos y no frágil si no cumple ninguno de ellos. ${ }^{8}$ Se estima que $15 \%$ de los adultos no institucionalizados en los Estados Unidos son frágiles y las estimaciones globales de fragilidad oscilan entre 3.5 y $27.3 \% .^{10}$

Se han utilizado diferentes pruebas para lograr identificar a pacientes con este síndrome. Uno de los más importantes es el índice de fragilidad de Rockwood, el cual consta de 70 preguntas relacionadas con el historial del paciente, tomando en cuenta comorbilidades, actividades diarias, estado de ánimo, funcionalidad y estado nutricional. Este índice no requiere un examen físico, lo que es importante en pacientes con patologías agudas y/o graves, evita realizar pruebas físicas o de caminata y se puede obtener de un familiar cercano. ${ }^{6,11}$ Una de sus desventajas es la necesidad de obtener valores de laboratorio como biometría hemática y química sanguínea, además es ineficiente realizarlo en el servicio de urgencias por su gran cantidad de preguntas. Por el contrario, el índice de FRAIL (por sus siglas en inglés: Fatigue Resistance, Aerobic, Illnesses, Loss of weight) es una prueba validada y recomendada como cribado para identificar personas frágiles, el cual consiste en cinco preguntas fáciles de contestar y que igualmente no ameritan un examen físico y se puede obtener de un familiar cercano. ${ }^{6}$

En un estudio retrospectivo que incluyó 432,828 pacientes realizado por el grupo de Myrick C. Shinall Jr., se identificó que los pacientes frágiles y muy frágiles presentaban una tasa de mortalidad a 30 días muy elevados en cirugías consideradas como de menor estrés $10.34 \%$ (IC $95 \%, 7.73-13.48 \%$ ) y en procedimientos quirúrgicos de

Tabla 4: Regresión logística univariada y multivariada de riesgo de complicaciones mayores.

Complicaciones mayores

Univariado

\begin{tabular}{|c|c|c|c|c|c|c|}
\hline \multirow[b]{2}{*}{ Variable } & \multirow[b]{2}{*}{ OR } & & & & & \\
\hline & & IC 95\% & $\mathrm{p}^{*}$ & OR & IC 95\% & $p^{*}$ \\
\hline Edad & 1.018 & 0.93 a 1.10 & 0.692 & - & - & - \\
\hline Sexo & 0.601 & 0.195 a 1.85 & 0.377 & - & - & - \\
\hline Fragilidad & 4.479 & 1.13 a 17.73 & 0.033 & 4.813 & 1.265 a 18.314 & 0.021 \\
\hline
\end{tabular}


Figura 1: Comparación de medianas para test $\mathrm{CCl}$ entre ambos grupos.

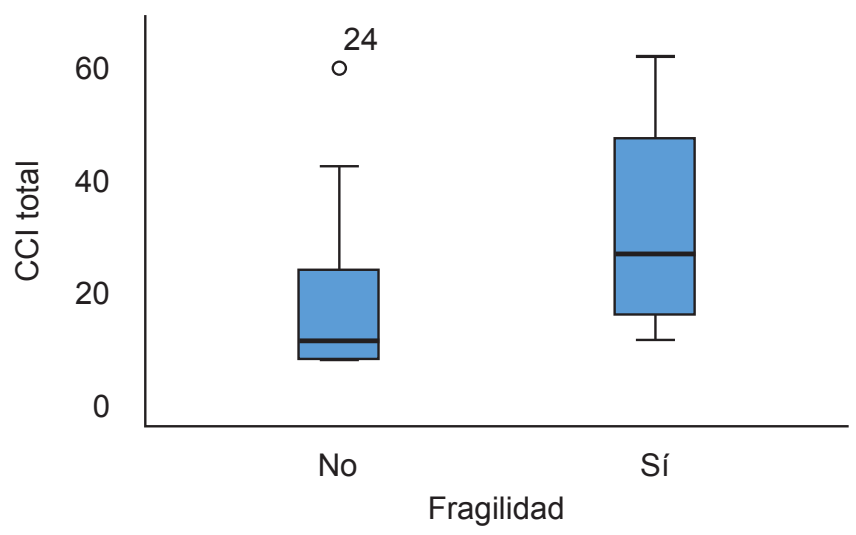

Figura 2: Comparación de medianas para edad entre ambos grupos.

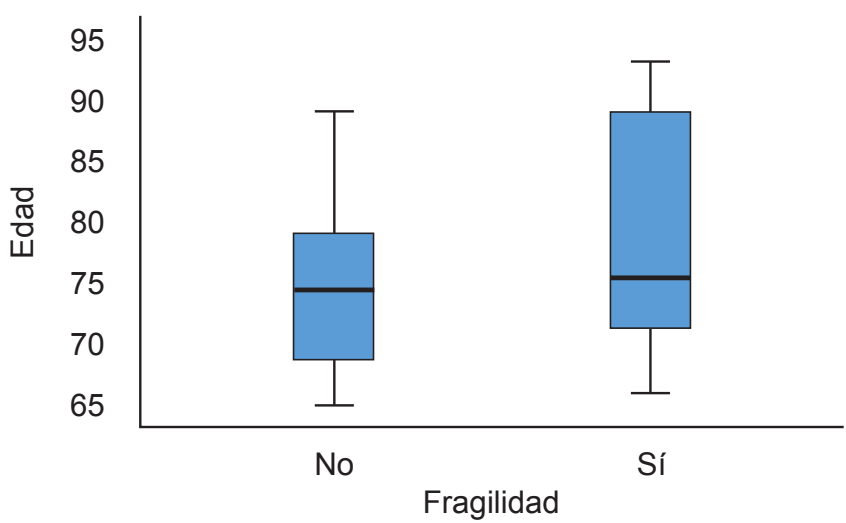

estrés moderado 18.74\% (IC 95\%, 17.72-19.80\%). La mortalidad aumentó a los 90 y 180 días de postquirúrgicos hasta casi 50\% (43.00\%; IC 95\%, 4.69-44.32\%). En nuestro estudio no fue posible identificar diferencias clínicas y estadísticamente significativas para la mortalidad 1 vs. 0; $p=0.217$ (IC 95\% 21.581 a 38.136). ${ }^{12}$

Recientemente en un estudio realizado por Giuseppe y colaboradores que incluyó 11,258 pacientes con nefrectomía radical, se observó que los pacientes frágiles mostraron tasas más elevadas de complicaciones generales $62.6 \%$ vs. $50.9 \%$ (OR 1.46), mortalidad hospitalaria $1.6 \%$ vs. $1.0 \%$ (OR 1.52) y mayor estancia hospitalaria (seis frente a un día, OR 1.36). De igual forma el grupo de Bellal Joseph identificó que en pacientes frágiles con trauma tenían 33.4\% más probabilidades de desarrollar complicaciones hospitalarias $(\mathrm{p}=0.02)$ así como mayor número de reingresos (odds ratio [OR], 1.4; IC 95\%, 1.2-3.6) y mayor mortalidad (OR 1.1; IC del 95\%, 1.04-4.7) en comparación con los pacientes no frágiles. En otro estudio realizado por el mismo autor que inclúa 220 pacientes postoperados, se utilizó el índice de
Rockwood para diferenciar a pacientes frágiles de los no frágiles, se identificó que los pacientes frágiles tienen un riesgo 3.87 veces más elevado de presentar complicaciones mayores (OR [95\% IC]: 3.87 [1.69-8.84], $p=0.001) .{ }^{11}$ En nuestro estudio observamos que los pacientes con fragilidad demostraron tener cuatro veces más riesgo de complicaciones (OR [95\% IC]: 4.479 [1.13 a 7.73], p = 0.033), entre las cuales destacó: más ingresos a la unidad de terapia intensiva en $38.5 \%$ de los casos ( $p=0.049$ ).

Una de las debilidades de nuestro estudio es el número de pacientes estudiado; a pesar de ello, obtuvimos resultados clínicos estadísticamente significativos para tomar en cuenta el índice de fragilidad FRAIL como predictor de complicaciones postquirúrgicas. Otra debilidad del estudio es que no tomamos en cuenta el estado en el que los pacientes ingresaron al servicio de urgencias, es decir, no fueron clasificados de acuerdo con la gravedad preoperatoria así como el tiempo de evolución. Partiendo de la teoría de que a mayor tiempo de evolución mayor probabilidad de complicaciones sería de gran relevancia evaluar a los pacientes con alguna prueba como POSSUM al ingreso a un servicio de urgencias y comparar con el estado postquirúrgico. De igual forma, no mencionamos el tipo de cirugía realizada, por lo que desconocemos si las complicaciones son consecuencia directa del tipo de cirugía o se trata de un resultado al azar. Es necesario realizar un estudio donde podamos analizar una muestra con un solo tipo de cirugía, tomando en cuenta las variables mencionadas para obtener un poder estadístico mucho mayor.

\section{CONCLUSIONES}

En nuestro estudio identificamos que el índice de FRAIL es una herramienta fácil de utilizar en un servicio de urgencias, ya que no es necesario realizar pruebas físicas, además clasifica de manera oportuna a quienes tienen síndrome de fragilidad. De la misma forma identificamos que los pacientes con índices mayores de 25 puntos tienen un riesgo cuatro veces mayor de complicaciones postquirúrgicas OR $=4.479(\mathrm{IC} 95 \% 1.13$ a 17.73, $\mathrm{p}=0.033)$.

\section{AGRADECIMIENTOS}

Al Servicio de Enseñanza del Hospital Ángeles Mocel y residentes de cirugía general.

\section{REFERENCIAS}

1. An aging nation: The older population in the United States; Currente population reports 2014. Current Population Reports Internet. Washington, DC: US Census Bureau, Population Projections Branch. Disponible en: https://www.census.gov/library/publications/2014/ demo/p25-1140.html

2. Base de datos INEGI [Internet]. Encuesta Nacional de la Dinámica Demográfica SNEGI; 2018. [Actualizado 30 de septiembre de 2019] 
Disponible en: https://www.inegi.org.mx/contenidos/saladeprensa/ aproposito/2019/edad2019_Nal.pdf

3. Kim SW, Han HS, Jung HW, Kim KI, Hwang DW, Kang SB et al. Multidimensional frailty score for the prediction of postoperative mortality risk. JAMA Surg. 2014; 149 (7): 633-640.

4. Joseph B, Zangbar B, Pandit V, Kulvatunyou N, Haider A, O'Keeffe T et al. Mortality after trauma laparotomy in geriatric patients. J Surg Res. 2014; 190 (2): 662-666.

5. Joseph B, Zangbar B, Pandit V, Fain M, Mohler MJ, Kulvatunyou N et al. Emergency general surgery in the elderly: too old or too frail? J Am Coll Surg. 2016; 222 (5): 805-813.

6. Fried LP, Tangen CM, Walston J, Newman AB, Hirsch C, Gottdiener J et al. Frailty in older adults: evidence for a phenotype. J Gerontol A Biol Sci Med Sci. 2001; 56 (3): M146-M156.

7. Chen X, Mao G, Leng SX. Frailty syndrome: an overview. Clin Interv Aging. 2014; 9: 433-441.
8. Morley JE, Vellas B, van Kan GA, Anker SD, Bauer JM, Bernabei R et al. Frailty consensus: a call to action. J Am Med Dir Assoc. 2013; 14 (6): 392-397.

9. Saucedo-Moreno EM, Fenig-Rodríguez J. Estadística en cirugía, cómo entender y aplicar conceptos básicos. Cir Cir. 2019; 87 (6): 692-697.

10. Bandeen-Roche K, Seplaki CL, Huang J, Buta B, Kalyani RR, Varadhan $\mathrm{R}$ et al. Frailty in older adults: a nationally representative profile in the United States. J Gerontol A Biol Sci Med Sci. 2015; 70 (11): 1427-1434.

11. Rockwood K, Andrew M, Mitnitski A. A comparison of two approaches to measuring frailty in elderly people. J Gerontol A Biol Sci Med Sci. 2007; 62 (7): 738-743.

12. Rockwood K, Mitnitski A, Song X, Steen B, Skoog I. Long-term risks of death and institutionalization of elderly people in relation to deficit accumulation at age 70. J Am Geriatr Soc. 2006; 54 (6): 975-979. 